\title{
Dynamics of Wetting Fronts in Porous Media
}

\author{
Igor Mitkov \\ Center for Nonlinear Studies and Computational Science Methods Group \\ Los Alamos National Laboratory, Los Alamos, NM 87545 \\ Daniel M. Tartakovsky and C. Larrabee Winter \\ Geoanalysis Group, Earth and Environmental Science Division \\ Los Alamos National Laboratory, Los Alamos, NM 87545
}

(November 10, 2018)

\begin{abstract}
We propose a new phenomenological approach for describing the dynamics of wetting front propagation in porous media. Unlike traditional models, the proposed approach is based on dynamic nature of the relation between capillary pressure and medium saturation. We choose a modified phase-field model of solidification as a particular case of such dynamic relation. We show that in the traveling wave regime the results obtained from our approach reproduce those derived from the standard model of flow in porous media. In more general case, the proposed approach reveals the dependence of front dynamics upon the flow regime.

PACS: 47.55.Mh, 87.22.As, 47.55.Kf, 68.35.Ja
\end{abstract}

The dynamics of fluids in porous media has been a subject of numerous theoretical and experimental studies because of its importance for engineering and environmental applications [1] 3]. Among the most challenging problems in this area is modeling fluid flow through a partially saturated medium, in particular the propagation of wetting (or drying) fronts. This has been addressed on both "microscopic" (or pore-scale) and "macroscopic" (on scales larger than pore size) levels. The fluid dynamics in the pore networks has been studied by numerous authors both theoretically [4] and experimentally [5,6. These studies on the microscopic scale provide valuable insight into the underlying mechanisms of fluid transport in porous media. A microscopic description requires the detailed information of the pore structure and pore-size distribution. When this information is not available, as often happens for large domains, one has to rely upon a macroscopic description. This description is useful for determining such integral characteristics, as the width and propagation velocity of moving wetting fronts, the influence of its curvature on the dynamics, etc.

The subject of the present work is the dynamics of wetting fronts in porous media when a liquid phase (water) displaces air. A straightforward description of this process consists of treating wetting fronts as sharp interfaces which separates completely wet and dry regions [5,7]. However, in many realistic cases the structure of the transitional zone (wherein water saturation varies gradually) cannot be neglected. One can either describe the dynamics of the liquid and air phases separately, or noting that air pressure is close to atmospheric, consider only the dynamics of the liquid phase. These two approaches generally produce similar results (Ref. [8], p. 213). The latter approach seems to be more attractive due to its simplicity.

Within the framework of the chosen approach wa- ter flow is described by Darcy's law which, similarly to Ohm's law for electric current, stipulates the proportionality between flux and gradient of a potential (see, e.g., [7]). For flow through porous media a water pressure, averaged over many pore sizes, plays the role of the potential. Richards [9] has empirically generalized Darcy's law onto flow in partially saturated porous media (PSPM) by letting the proportionality coefficient depend on saturation $\theta$ of the medium $(0<\theta<1)$. Coupled with the mass conservation law, the generalized Darcy's law constitutes the existing macroscopic description of the fluid dynamics in PSPM. To complete this description, one needs to specify a relation between $\theta$ and capillary pressure (normalized by the product of fluid density $\rho$ and gravitational acceleration $g$ ), $\psi$. Traditionally this relation is assumed to be algebraic [1, 10]. However, this contradicts the numerous experimental evidences revealing the dependence of $\theta-\psi$ relation upon the conditions of the experiment. In particular, this relation exhibits hysteresis for wetting and drying of a medium [1, 10, 11 .

In the present Letter we propose a novel phenomenological approach to describe propagation of wetting fronts in porous media, which is based on a dynamic $\theta-\psi$ relation. Our description consists of two dynamic equations for $\theta$ and $\psi$ coupled by nonlinear sources. This implies that the traditional algebraic $\theta-\psi$ correspondence is replaced by a nonlocal (integro-differential) relationship. The proposed model is a new application of the well-known "phase-field" approach used to describe solidification, electro-deposition, and other physical problems [12 17]. We show that, under certain conditions, dynamics of the wetting front in our approach reproduce that in the traditional approach. The same is true for the pressure profiles associated with the wetting fronts obtained from both models. We demonstrate that, under different conditions, this equivalence breaks down, with 
our model revealing a dynamic nature of $\theta-\psi$ relation.

The generalized Darcy's law for flux q through PSPM has a form $\mathbf{q}=-K(\theta) \nabla\left(\psi-x_{3}\right)$, where $K$ is the saturation-dependent conductivity of the medium and $x_{3}$ is the vertical coordinate (positive downward) that stands for the gravitational component of pressure (normalized by $\rho g$ ). Here $\psi$ is measured relative to the ambient atmospheric pressure, so that $\psi<0$ for partially saturated and $\psi \geq 0$ for fully saturated medium. Combined with the mass conservation law, $\partial \theta / \partial t=-\nabla \cdot \mathbf{q}$, this leads to the equation introduced by Richards [9]

$$
\frac{\partial \theta}{\partial t}=\nabla \cdot\left[K(\theta) \nabla\left(\psi-x_{3}\right)\right] .
$$

Over the years a number of algebraic $\theta-\psi$ and $K(\theta)$ relations have been proposed empirically (see, e.g. [18 20]). Richards equation (RE) (1) combined with such a relation constitutes the traditional approach to describe flow in PSPM. Since algebraic relations are not supported by experimental data, in what follows we propose a new approach with a dynamic $\theta-\psi$ relation.

To describe flow in PSPM, we modify the phase-field model (PFM) from 12]

$$
\begin{aligned}
\frac{\partial \psi}{\partial t} & =D \nabla^{2} \psi-\frac{1}{S} \frac{\partial \theta}{\partial t}-D \frac{\partial \theta}{\partial x_{3}} \\
\tau \frac{\partial \theta}{\partial t} & =W^{2} \nabla^{2} \theta \\
& +\left[2 \theta-1-\lambda\left(\psi-\psi_{f}\right) \theta(1-\theta)\right] \theta(1-\theta),
\end{aligned}
$$

where $D=K_{s} / S$ is the diffusion coefficient, $K_{s}$ is the conductivity of a fully saturated medium, $S$ is the specific storage (measure of compressibility of the fluid and medium), $\tau$ is a characteristic time-scale of the saturation dynamics, and $W$ is the width of a moving wetting front. The model parameter $\lambda$ will be determined as a function of macroscopic parameters $K_{s}, W$ and $\tau$. The constant $\psi_{f}$ is the normalized capillary pressure along the moving front in the sharp-interface limit. We will demonstrate below that $\psi_{f}$ coincides with the pressure in the dry medium far ahead of the wetting front. Since the width of the capillary zone, associated with the localized wetting fronts, is much smaller than the typical scale of the pressure variation, the following holds $W^{2} / \tau \ll D$. We have added the last term in (2) to PFM [12], to account for the gravitational force.

Our model is phenomenological in the sense that presently we do not provide a rigorous physical motivation for the nonlinear source term on the right-hand-side of (3). Nevertheless, the model captures the main features of the wetting fronts propagation in porous media. In particular, numerous experiments [21,22 have shown that, under certain conditions, the wetting fronts remain localized and propagate in a self-similar manner. The medium is fully saturated $(\theta=1)$ behind the front region and completely dry $(\theta=0)$ ahead of the front. The cubic polynomial in (3) provides for such a structure of the wetting front (similar to the PFM for solidification). The fact that the liquid moves in the direction opposite to the pressure gradient is accounted for by the term proportional to $\left(\psi-\psi_{f}\right)$ in (3), since its presence makes the depths of the two minima of the corresponding potential energy different.

Eqs. (2) -(3) have to be supplemented by a constraint to ensure conservation of mass. The specific storage is related to the compressibility of fluid and porous medium as $S=\rho g \omega\left(\beta_{f}-\beta_{s}+\beta_{p}\right)$ (Ref. [8], p. 108). Here $\omega$ is the medium porosity (fraction of pore volume in the total volume $v$ of the medium) and $\beta_{f}, \beta_{m}$, and $\beta_{p}$ are the compressibility coefficients of fluid, solid grains, and pores, respectively. The total mass of the fluid is given by $M=\int_{v} \omega \rho \theta d \mathbf{x}$. For incompressible fluids and media $(S=0)$, since $D=K_{s} / S$, Eq. (2) reads $\partial \theta / \partial t=K_{s}\left(\nabla^{2} \psi-\partial \theta / \partial x_{3}\right)$, which conserves mass. For compressible fluids and media $d M / d t=Q$, where $Q$ is the total mass flux through the medium, provides the global constraint on the system (2)-(3).

Since $S$ is the specific storage of a medium, the effect of compressibility of fluids and media on flow dynamics is characterized by the dimensionless parameter $S L$, where $L$ is a typical domain size. For many practical applications, such as flow of water through low porosity rocks, $S \sim 10^{-5}-10^{-7} \mathrm{~m}^{-1}$ and $L \sim 10^{-1}-10^{3} \mathrm{~m}$. Thus $S L \ll 1$, and the fluid and medium are virtually incompressible.

We consider propagation of one-dimensional (1D) wetting fronts in a slightly compressible medium with $S L \ll$ 1. The dynamics of these fronts is described by 1D Eqs. (2) $-(3)$, and the mass conservation constraint is satisfied automatically. Two different situations exist: horizontal (gravity-free) and vertical front propagation. To maintain propagation of a self-similar front, we choose the constant flux condition $\partial\left(\psi-x_{3}\right) / \partial x_{i}=-q / K_{s}$ at $x_{i}=0$, and no-flux condition for capillary force $\partial \psi / \partial x_{i}=0$ at $x_{i}=L$. Here $i=1$ or $i=3$ for horizontal or vertical flow, respectively, and $q$ is an external velocity flux. At the boundary behind the front, $x_{i}=0$, the medium is fully saturated $(\theta=1)$, and at the boundary ahead of the front, $x_{i}=L$, the medium is dry $(\theta=0)$.

Let us apply a traveling wave ansatz $z=x_{i}-V t$, for a front moving with velocity $V$, to $1 \mathrm{D}$ Eqs. (2)-(3). Relations $W \sim V \tau$ and $W^{2} / \tau \ll D$ give rise to a small parameter $P e \ll 1$, where $P e=V W / D$ is the Péclet number. The perturbation analysis around $P e=0$, similar to that performed in 12], gives a front moving with velocity $V=q$, and saturation and capillary pressure profiles

$$
\begin{aligned}
& \theta(z)=\frac{1}{2}\left[1-\tanh \left(\frac{z}{2 \sqrt{2} W}\right)\right]+O(P e), \\
& \psi(z)=\psi_{f}+J\left\{W \sqrt{2} \ln 2-\frac{z}{2}\right.
\end{aligned}
$$




$$
\left.+W \sqrt{2} \ln \left[\cosh \left(\frac{z}{2 \sqrt{2} W}\right)\right]\right\}+O\left(P e^{2}\right) .
$$

Here $J$ is an absolute value of the capillary pressure gradient at the boundary. It is given by $J=q / K_{s}$ for a horizontally propagating front, and by $J=\left(q / K_{s}-1\right)$ for a vertically propagating front. A vertical wetting front does not exist when $q \leq K_{s}$, i.e. when the external flux is not large enough to compensate the effect of the gravity force, and thus develop a saturated zone.

The saturation profile (14) is evaluated in the zeroth order in $P e$, since for the localized wetting fronts saturation varies in the narrow region $W \ll \sqrt{D \tau}$. Hence the higher order corrections to $\theta$ influence the nonlocal pressure profile (5) only in the second order. The solvability condition for the equation for $\theta$ in the first order (similar to [12]) yields $\tau / \lambda \approx-0.313 W^{2} J / q$.
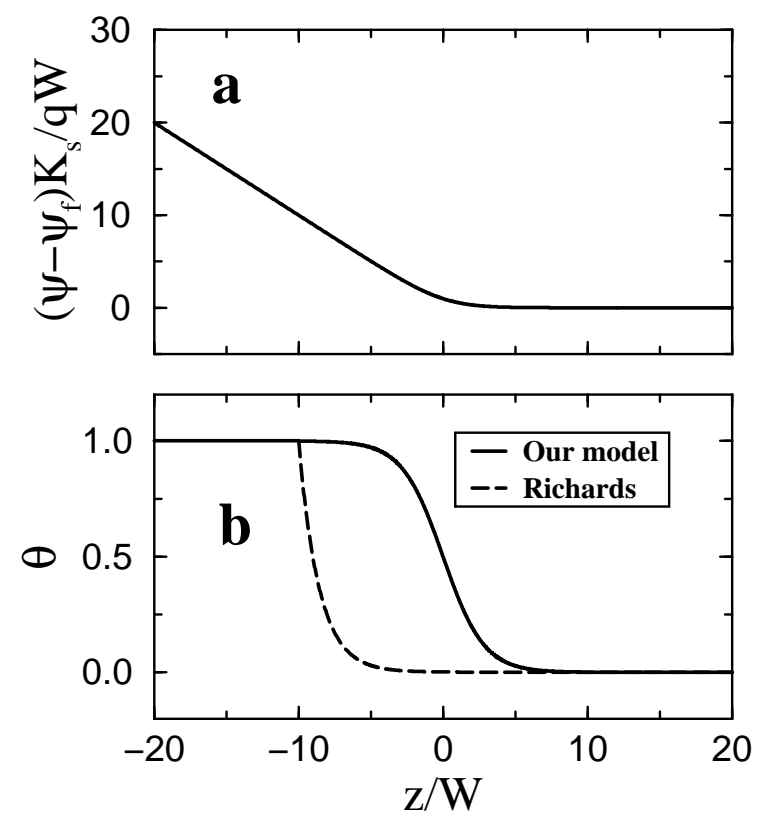

FIG. 1. Normalized capillary pressure $\left(\psi-\psi_{f}\right) K_{s} / q W$ (a) and saturation (b) profiles in traveling wave coordinate. In (b) normalized capillary pressure in a dry medium is taken $\psi_{f} K_{s} / q W=-10$.

We now compare our approach with the traditional (Richards) approach. One of the most widely-used examples of algebraic functions $\theta(\psi)$ and $K(\theta)$, complementary to RE (1), has been proposed by Gardner [10, 18]

$$
\theta(\psi)=e^{\alpha \psi}, \quad K(\theta)=K_{s} \theta,
$$

where $\alpha$ is the pore-size distribution parameter. The first equation in (6) is valid for $\psi<0$ (when the medium is partially saturated), while for $\psi \geq 0$ (for a fully saturated medium) $\theta \equiv 1$. The same boundary conditions as before are used. Substituting (6) into 1D Eq. (11) and applying the traveling wave ansatz gives, after a series of transformations, the solution for the pressure profile

$$
\psi(z)=\frac{\ln 2}{\alpha}+\psi_{L}-\frac{J z}{2}+\frac{1}{\alpha} \ln \left[\cosh \left(\frac{\alpha J}{2} z\right)\right],
$$

where $\psi_{L}$ is a large negative number corresponding to the pressure in the dry medium far ahead of the front.

Comparing (7) with (5) we find that the pressure profiles obtained from both models coincide, provided the relations between parameters $\sqrt{2} W J=\alpha^{-1}$ and $\psi_{f}=\psi_{L}$ hold. Figure $\mathbb{1}$ (a) shows the resulting pressure profile from both models. Substituting (7) into the first relation in (6) we obtain the saturation profile for Richards model. The result is compared with the saturation profile (堛) in the Figure 11(b). Although the saturation profiles differ within the capillary zone, this difference occurs on the very small scale $W$. An unphysical sharp kink that appears in the Richards model for $\theta(z)$ is absent in our model. Comparing Figure 1 (b) with the experimental data presented on Figures 2-5 of Ref. 22] shows that the saturation profile obtained by our model agrees with the experiments.

Though in the traveling wave regime our model reproduces the results obtained from the Richards model, it is also capable of capturing the dynamic $\theta-\psi$ relation in different regimes, without adjusting the parameters. This is not the case for the Richards model, where one needs to adjust the parameter $\alpha$ in (6) to different experimental regimes. To demonstrate the dynamic nature of the $\theta-\psi$ relation in our model, we have performed the numerical simulations of $1 \mathrm{D}$ Eqs. (2) $-(3)$ under several boundary conditions. We solved system (2)-(3) using finite differences. Note that though in our simulations $S L \ll 1$, we keep the term $S \partial \psi / \partial t$ in (2). Despite being small, this term provides relaxational feature to numerical solution of (2), which stabilizes the numerical algorithm. Figure 2 shows $\theta(\psi)$ obtained from these simulations for horizontal flow. The solid line in the figure corresponds to the traveling wave regime, resulting from the previously described boundary conditions. The dashed line represents $\theta(\psi)$ at time $t=1000$ corresponding to the wetting with the constant pressure at $x_{1}=0$ and the same no-flux condition at $x_{1}=L$.

Note that in the limit of the narrow capillary zone, $W \rightarrow 0$, our model describes the dynamics of sharp interface which separates completely wet $(\theta=1)$ and completely dry $(\theta=0)$ regions. This is in analogy with the PFM of solidification that reduces to the freeboundary problem [12]. It follows from (5) that in this limit $\psi(0)=\psi_{f}$ and $\psi_{z}(0)=-J$. Moreover, (2) reduces to a diffusion equation which is commonly used to describe flow in saturated media.

In conclusion, we have proposed a novel approach to describe dynamics of wetting fronts in porous media. Unlike the traditional approach, our phenomenological approach reflects the dynamic nature of the relation between capillary pressure and saturation of a medium. We have found that this relation varies with the flow 
regimes, which is supported by experimental data. We have demonstrated that, in the traveling wave regime, the proposed model reproduces the results obtained from the standard approach. We plan to extend our novel approach to two- and three-dimensional media incorporating in the description the front curvature and anisotropy of the medium. We expect to develop experimental support for the proposed approach by measuring quantitative features of the wetting fronts in porous media, such as the dependence of the front width on external flux.

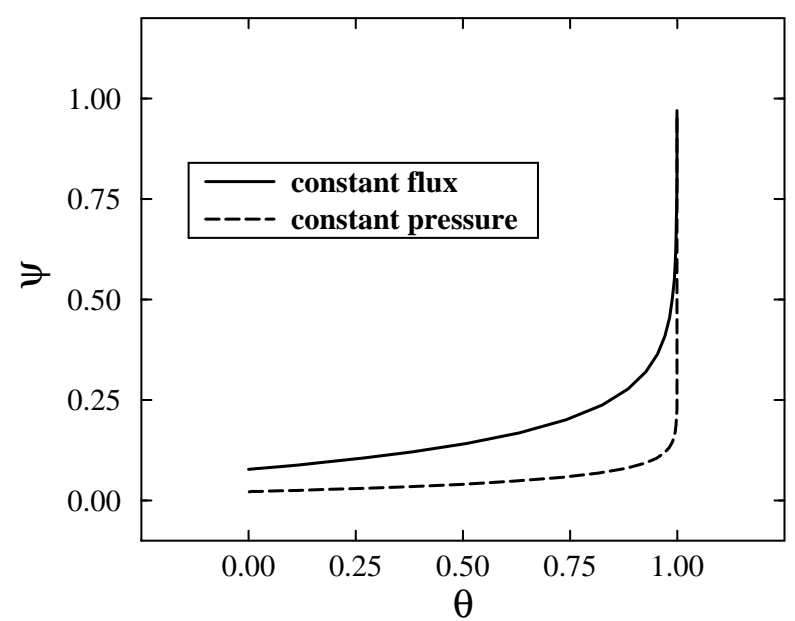

FIG. 2. Dynamic $\theta-\psi$ relation for both traveling wave and non-traveling wave regime. Parameters of the simulations are $D=1, W=0.1, S=0.005, \tau=1$. For the traveling wave regime $q=K_{s}$. Value of $\lambda$ is calculated according to the selection result $\tau / \lambda \approx-0.313 W^{2} J / q$. System size $L=10$ and number of grid-points is 201. Time step $d t=0.001$.

We are grateful to R. Camassa, G. Forest, M. Hyman, S.-Y. Chen, and J. Glazier for fruitful and clarifying discussions.

[1] M. Sahimi, Rev. Mod. Phys. 65, 1393 (1993); M. Sahimi, Flow and Transport in Porous Media and Fractured Rock (VCH, Weinheim, Germany, 1995).

[2] G. I. Barenblatt, V. M. Entov, and V. M. Ryzhik, Theory of fluid flows through natural rocks (Kluwer Academic Publishers, Boston, 1990).

[3] A. E. Scheidegger, The Physics of Flow Through Porous Media (University of Toronto Press, 1974).

[4] P. Collet, J. De Coninck, F. Dunlop, and A. Regnard, Phys. Rev. Lett. 79, 3704 (1997); A.-L. Barabási, Phys. Rev. Lett. 76, 3750 (1996); C. J. Boulter and A. O. Parry, Phys. Rev. Lett. 74, 3403 (1995); L. M. Schwartz, N. Martys, D. P. Bentz, E. J. Garboczi, and S. Torquato, Phys. Rev. E 48, 4584 (1993).

[5] R. Lenormand, E. Touboul, and C. Zarcone, J. Fluid Mech. 189, 165 (1988).
[6] J. Crassous, E. Charlaix, and J.-L. Loubet, Phys. Rev. Lett. 78, 2425 (1997); T. Delker, D. B. Pengra, and P.-z. Wong, Phys. Rev. Lett. 76, 2902 (1996); M. A. Knackstedt, B. W. Ninham, and M. Monduzzi, Phys. Rev. Lett. 75, 653 (1995); C. Du, B. Xu, Y.C. Yortsos, M. Chaouche, N. Rakotomalala, and D. Salin, Phys. Rev. Lett. 74, 694 (1995).

[7] L. P. Kadanoff, J. Stat. Phys. 39, 267 (1985).

[8] G. de Marsily, Quantitative Hydrogeology, (Academic Press, San Diego, 1986).

[9] L. A. Richards, Physics, 1, 318-333, 1931.

[10] J. Bear, Dynamics of fluids in porous media (Dover Publications, Inc., New York, 1972).

[11] E. E. Miller and R. D. Miller, J. Applied Phys. 27, 324 (1956).

[12] A. Karma and W.-J. Rappel, Phys. Rev. E 53, R3017 (1996).

[13] A. Karma and W.-J. Rappel, Phys. Rev. Lett. 77, 4050 (1996).

[14] J. S. Langer, Directions in Condensed Matter (World Scientific, Singapore, 1986), p. 164.

[15] J. B. Collins and H. Levine, Phys. Rev. B 31, 6119 (1985).

[16] R. Kobayashi, Physica D 63, 410 (1993).

[17] S.-L. Wang and R. F. Sekerka, Phys. Rev. E 53, 3760 (1996).

[18] W. R. Gardner, Soil Sci. 85, 223 (1958).

[19] M. T. van Genuchten, Soil Sci. Soc. of Am. 44, 892 (1980).

[20] Y. Mualem, Water Resours. Res. 12, 513 (1976).

[21] G. Vachaud and J.-L. Thony, Water Resours. Res. 7, 111 (1971); G. Segol, Classic Groundwater Simulations: Proving and Improving Numerical Models (PTR Prentice Hall, Englewood Cliffs, New Jersey, 1994).

[22] R. Haverkamp, M. Vauclin, J. Touma, P. J. Wierenga, and G. Vachaud, Soil Sci. Soc. Am. J. 41, 285 (1977). 